\title{
LONG-TERM PREDICTIONS FOR HIGHLY ECCENTRIC ORBITS
}

\author{
José M. Ferrándiz, M. Eugenia Sansaturio and Jesús Vigo \\ Departamento de Matemática Aplicada a la Ingeniería, E.T.S. de Ingenieros \\ Industriales, Universidad de Valladolid, 47011 Valladolid, Spain
}

\section{ABSTRACT}

Predictability in orbital behaviour of artificial satel1ites depends on several factors: the accuracy required, the particular dynamical models formulated, the sets of variables chosen to describe them, the numerical or analytical techniques used and, specially, the specific trajectories to be established. In this paper we address the problem of predictability for highly eccentric satellites with $\left(J_{2}+J_{22}\right)$-perturbation, by using numerical techniques to integrate the equations of motion when expressed in different sets of regular variables.

\section{INT RODUCT ION}

The predictions of the orbital motion of an artificial satellite involves a great difficulty which comes not only from the fact that the real problem is indeed complicated but al so from the complexity of the mathematical model chosen to represent it. In particular, this turns out to be specially evident when we deal with satellites whose orbit has high eccentricity, say 0.9 or more. In these cases, the usual treatments of the problem can hardly provide the required accuracy even for the simplest force models. That is special1y true when we aim to obtain long-term predictions, which turn out to be particulary bad at the perigee.

In the 1 ight of these facts, and in order to manage with this kind of problems, we have to think of using either nonstandard numerical methods to integrate the equations of motion, or special sets of variables providing better conditioned equations for the numerical integration through standard codes. 
As for the variables.we can use, we find several sets among which we can mention here the universal variables, widely used by NASA, and the so-called regularizing ones, which include as a particular case the linearization methods, either in the version of coordinates or in that of elements.

Just to remind the reader, let us say that the 1 inearization methods provide harmonic oscillator form to the equations of motion with the advantages it involves when we integrate them numerically, such as the favorable propagation of the truncation error. On the other hand, the aim of the methods of elements is that the transformed dependent variables are constant or linear functions of the independent variable along a Keplerian motion.

It is controversial to say which set of variables is the best, if there is one. However, in the loght of our experience, we would say that regularizing variables behave better than the non-regularizing ones since the latter require more evaluations of derivatives and computing time to get a prescribed accuracy, by numerical integration, and give rise to a faster growth of numerical errors. All these facts favour regularization methods, specially when we are involved in long-term predictions.

In what follows we will concentrate on the comparison of the results obtained when using Cartesian coordinates and other two sets of regularizing variables: the KustaanheimoStiefel (KS) variables and the Burdet-Ferrandiz (BF) ones.

KS transformation and the time element associated with them are widely described in the well known book by Stiefel and Scheifele (1971) and we will only remind the reader that it reduces the Kepler motion to four harmonic oscillators with all the frequencies being equal to one, by introducing the energy integral into the equations of motion and using the eccentric anomaly as independent variable.

As for $B F$ variables, they are a set of 8 redundant canonical variables, the coordinates being the direction cosines of the particle and the inverse of its distance. They were first used by Burdet (1969) and reintroduced by Ferrandiz (1988), but in a Hamiltonian framework, obtaining equations of motion similar to ones derived by Burdet.These variables also allow us to reduce the Kepler problem to four harmonic oscillators with unit frequencies by introducing the integral of the angular momentum into the equations of motion and using the true anomaly as independent variable.

The homogeneous Hamiltonian of the problem when expressed in BF variables has the following expression

$$
\mathrm{cK}=\frac{1}{2}|\mathrm{p}|^{2}|\mathrm{x}|^{2}+\frac{1}{2} \mathrm{p}_{z}^{2} \mathrm{z}^{2}-\frac{\mu}{z}+\frac{\mathrm{p}_{\mathrm{o}}}{\mathrm{z}^{2}}+\mathrm{W}=0,
$$

where $x$ is the direction vector of the particle, $p$ stands 
for the conjugate momenta of $x, z=1 / r, w=z^{-2} V$ (V perturbing potentical), $c$ is the magnitude of the angular momentum and $\mathrm{p}_{\mathrm{O}}=-\mathrm{h}$ ( $\mathrm{h}$ energy).

The time element associated with $B F$ variables was introduced by the first two authors in a Spanish-Portuguese meeting held in June 89 , by defining two functions $e, E$ of the canonical $B F$ variables through the expressions

$$
e \sin E=\frac{\sqrt{2 p_{O}}}{\mu} z p_{z}=-\frac{\sqrt{2 p_{o}}}{\mu} \frac{c z^{\prime}}{z}, e \cos E=1-\frac{2 p_{o}}{\mu z} .
$$

The time element is thus defined by the generalized Kepler equation

$$
\ell-\ell_{0}=t-t_{0}-\frac{\mu}{\left(\sqrt{2 p_{0}}\right)^{3}}(E-s-e \sin E),
$$

s being the true anomaly.

Finally, the equation for the time element in conservative cases is given by

$$
\ell^{\prime}=\frac{\mu}{\left(\sqrt{2 p_{0}}\right)^{3}}+\frac{1}{c} \frac{\mu-\left(c^{2}+2 W\right) z}{\mu^{2}-2 p_{0}\left(c^{2}+2 W\right)} \frac{\partial W}{\partial z},
$$

where ()' stands for derivative with respect to s.

To obtain the corresponding equation for the time element in non-conservative cases it suffices to add up the following expression

$$
\begin{aligned}
{\left[\frac{3 \mu}{\left(\sqrt{2 p_{0}}\right)^{5}}(E-s-e \sin E)\right.} & -\frac{\mu}{\left(\sqrt{\left.2 p_{0}\right)^{3}} e \sin E\right.} \\
& \left.\times \frac{\mu+z\left(c^{2}+2 W\right)}{z\left\{\mu^{2}-2 p_{0}\left(c^{2}+2 W\right)\right\}}\right] \frac{\partial p_{0}}{\partial s},
\end{aligned}
$$

to the previous equation.

\section{RESULTS}

A first idea about the good behaviour of these sets of regularizing variables can be obtained from Ferrándiz et al. (1990) and from the results presented by the authors during the NATO ASI held in Cortina D'Ampezzo in August 90 . Such results are summarized in the plots shown in figures 1 to 5 , and refer to the problem of an artificial satellite with $\mathrm{J}_{2}$-perturbation whose orbit is equatoria1, has an eccenticity $\mathrm{e}^{2}=0.95$ and a perigee height of approximately 0.05 E.R. (300 $\mathrm{Km}$ ). However, we should point out that the results obtained 
with these data do not differ much from the ones obtained when we vary the inclination of the orbit in a wide strip around the equator and the location of the perigee.

In these plots we have used logarithmic scale in the vertical axis, representing

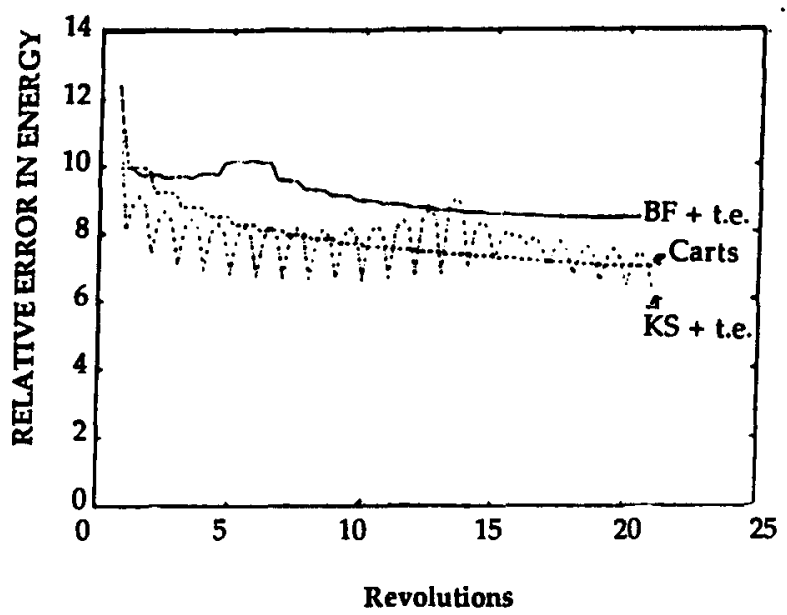

Figure 1

errors versus number of revolutions. There will appear two kinds of errors which will be referred to as

1. Trajectory error $\left(\varepsilon_{x}\right)$, which means the difference between the position given by a reference orbit, which we obtained by numerically integrating and requiring high enough accuracy, and the one obtained with the current numerical integration. Positions are compared for equal values of the independent variable, that is to say

$$
\varepsilon_{z}=\left|x_{\text {ref }}(S)-x_{\text {cal }}(S)\right|
$$

2. Position error $(\varepsilon)$, which apart from the previous error also includes an estimation of the error due to the integration of the equation of time $\left(\varepsilon_{t}\right)$. If we define $\left(\varepsilon_{t}\right)$ to be

$$
\varepsilon_{t}=\left|t_{\text {ref }}(S)-t_{c a l}(S)\right| . \mid \text { velocity } \mid \text {, }
$$

which means the 1 inear approximation of that error, the position error is then $\varepsilon=\varepsilon_{z}+\varepsilon_{t}$. 


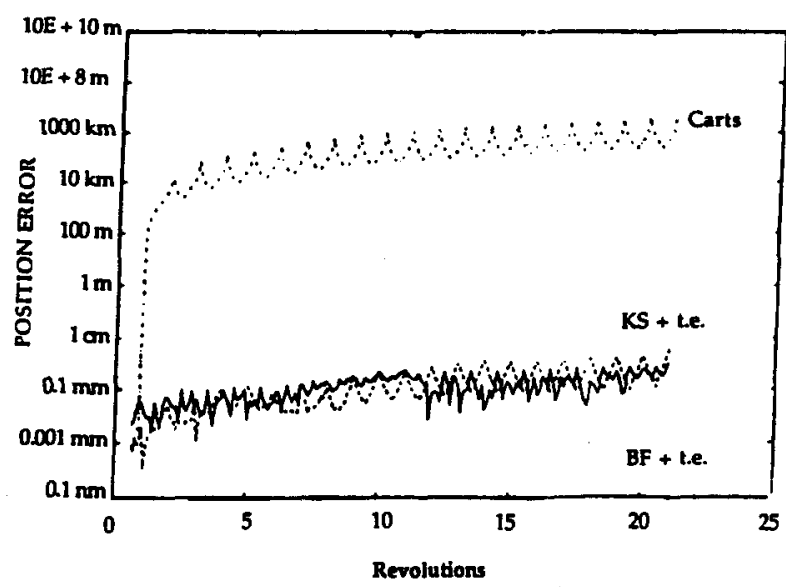

Figure 2

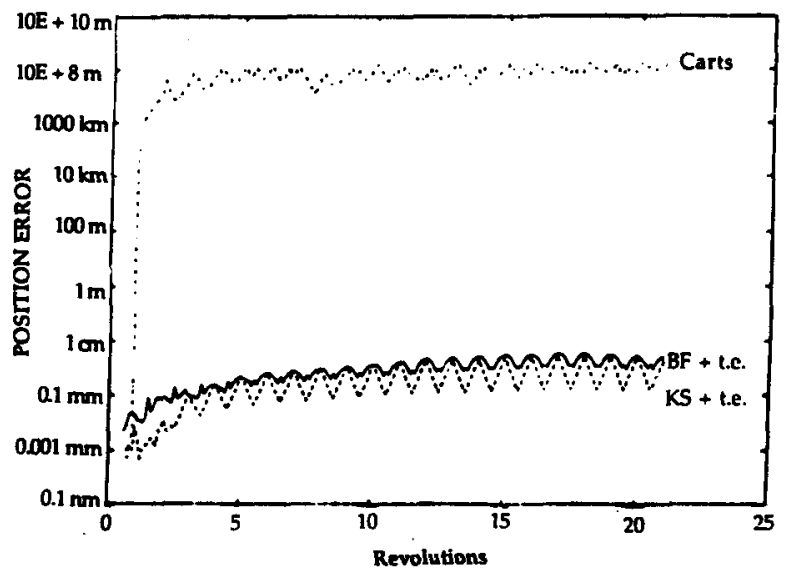

Figure 3

Figures 1 and 2 have been obtained by using a RungeKutta-Feh1berg (R-K-F) (7) 8 code, with variable stepsize, to integrate the equations when expressed in Cartesian coordinates (Carts.), KS coordinates (KSc.) and BF coordinates (BF) together with the equation of time. Although $B F$ coordinates provide a more accurate solution for the spatial coordinates (Fig. 1), the errors in the integration of the equation of time lead to similar results for the three sets of variables (Fig. 2). However, the computation of time from the corresponding elements gives rise to a considerably different behaviour, which turns out to be favourable for $B F+t . e$. (time element) (Fig. 3).

The use of regular elements associated with the KS transformation instead of the coordinates does not change their 


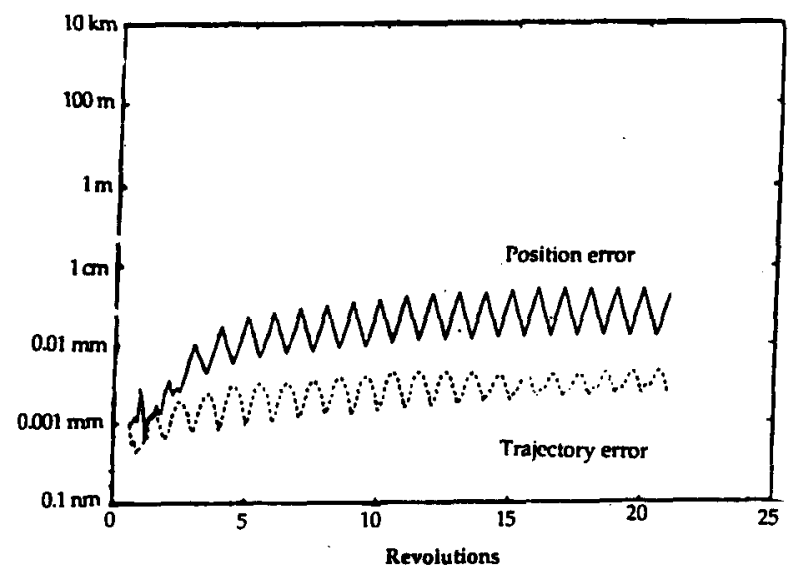

Figure 4 .

behaviour significantly when integrating very eccentric orbits (in accordance with Stiefel and Scheifele, p.95). Figure 4 shows the errors obtained by using: a) KS coordinates together with the equation of time (KSC. $+t), b) K S$ coordinates together with the equation of the time element (KSc.+t.e.), c) KS elements together with t.e. equation (KSe1.+t.e.) and choosing an Adams-Bashforth-Moulton (A-B-M) code of order 8 , with 144 steps per revolution.

This is not the case for BF coordinates, so Figure 5 clearly shows the improvement got when, in BF variables, we

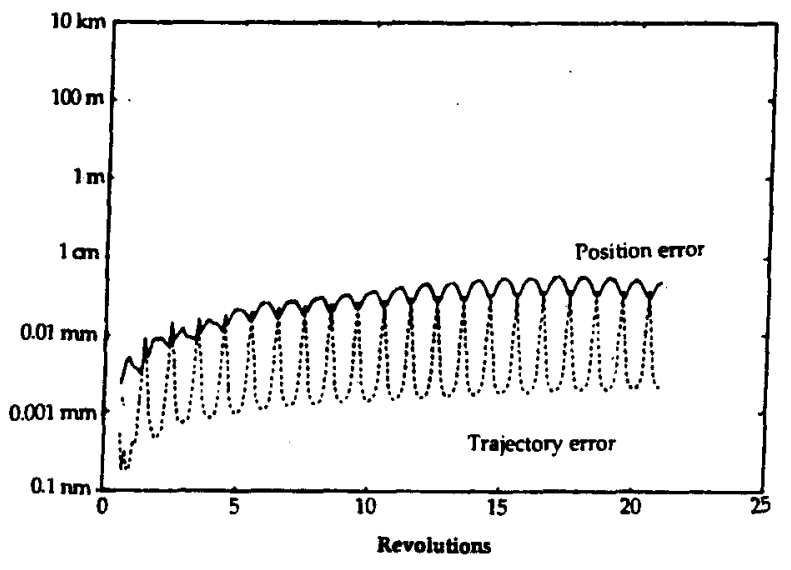

Figure 5. 
substitute the equation of time by the corresponding time element equation.

As a general comment, about the former results let us say that, for the same number of evaluations of derivatives, the use of BF variables together with their time element clearly improves the accuracy provided by KS methods: e.g. for A-B-M code the accuracy is improved in a factor greater or equal to $10^{2}$, reaching in some cases $10^{3}$.

The next step in our study consists in carrying out an analogous comparison but considering now that the perturbing

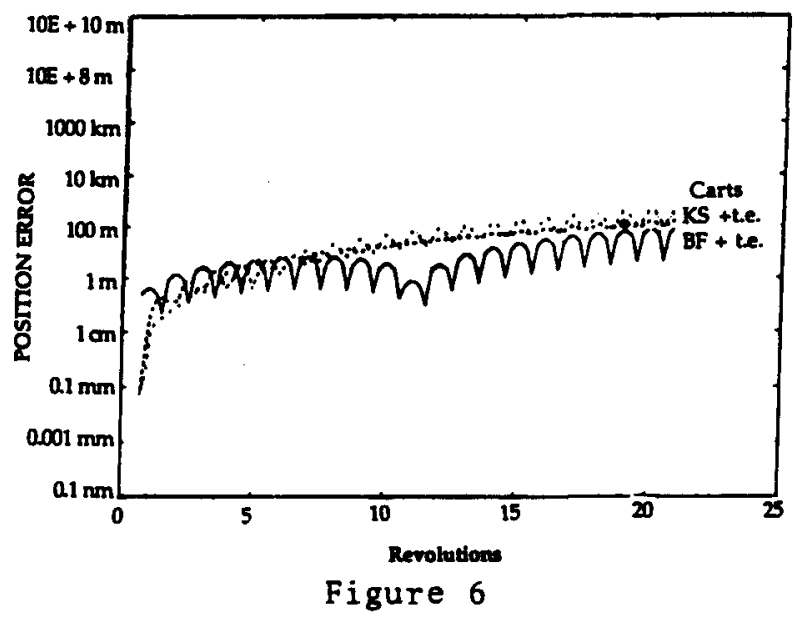

now that the perturbing potential also includes the $\mathrm{J}_{22}$-term. The results obtained in this case refer to a satellite with an eccentricity $e=0.9$, an inclination $I=10^{\circ}$ and a perigee of approximately $0.05 \mathrm{E} . \mathrm{R} .(300 \mathrm{Km}$. $)$.

By using an $R-K-F$ code to integrate the equations when

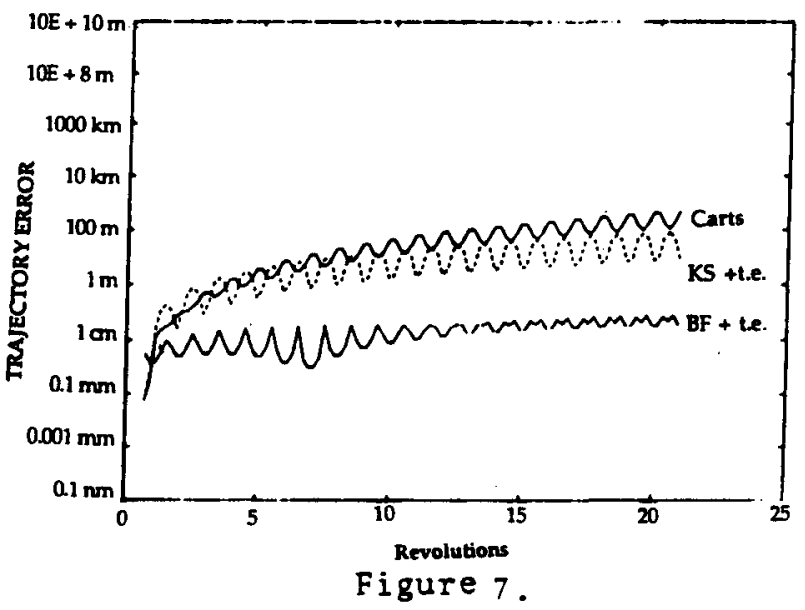


expressed in Cartesian coordinates, $\mathrm{KS}$ coordinates and $\mathrm{BF}$ coordinates together with their corresponding time element equation, figures 6 and 7 show trajectory and position errors, respectively, after 20 revolutions.

Again, BF coordinates provide a more accurate solution for the spatial coordinates (Fig. 6), whereas, in this case, the error in the integration of the equation of the time element lead to similar results for the three sets of variables, perhaps slightly more favorable for $B F+t . e$. (Fig. 7).

By means of an A-B-M code with 600 steps per revolution, figures 8 and 9 show the effect of the time error on the

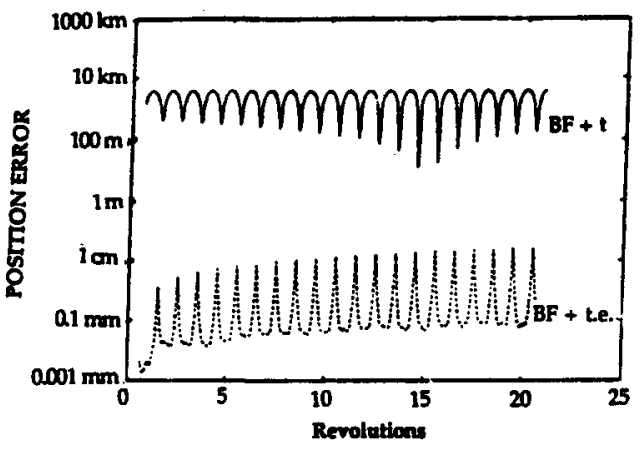

Figure 8

position error in more tetail, when we use BF coordinates (Fig. 8) and KS coordinates (Fig. 9). It is remarkable that the best accuracy for spatial coordinates - the minima of the trajectory error function - is got, for BF variables, at the perigee. This is not the case for KS variables since these points correspond to the maxima of the corresponding graphic of the trajectory error in figure 9 .

Figures 10 and 11 have. al-so been obtained,by using an A-B-M code with 600 and 1800 steps per revolution, respective$1 y$, to integrate the equations when expressed in Cartesian

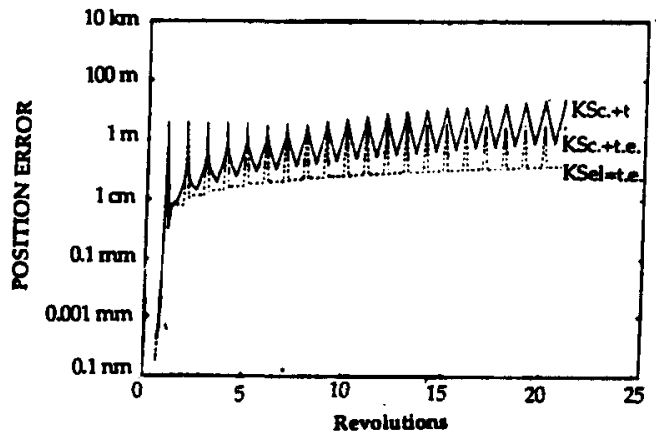

Figure 9 
coordinates, KS coordinates and BF coordinates together with their corresponding time element equation.

These plots clearly show the much worse behaviour of Cartesian coordinates with respect to the other two sets of regular variables, which behave in this case of $\left(\mathrm{J}_{2}+\mathrm{J}_{22}\right)$ perturbation in a similar way.

Fina11y, figure 12 represents the relative error in energy versus number of revolutions when using the above mentioned three sets of variables and an $R-K-F$ code has been chosen as integrator.

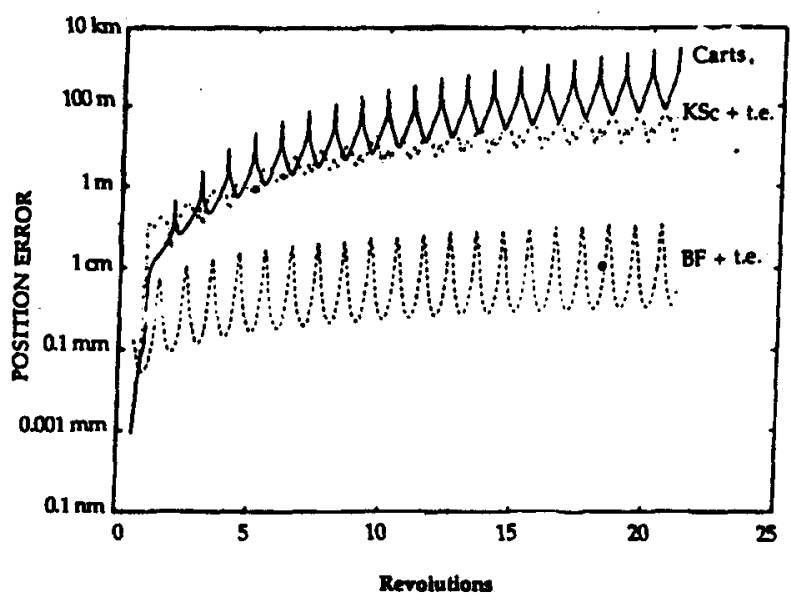

Figure 10 .

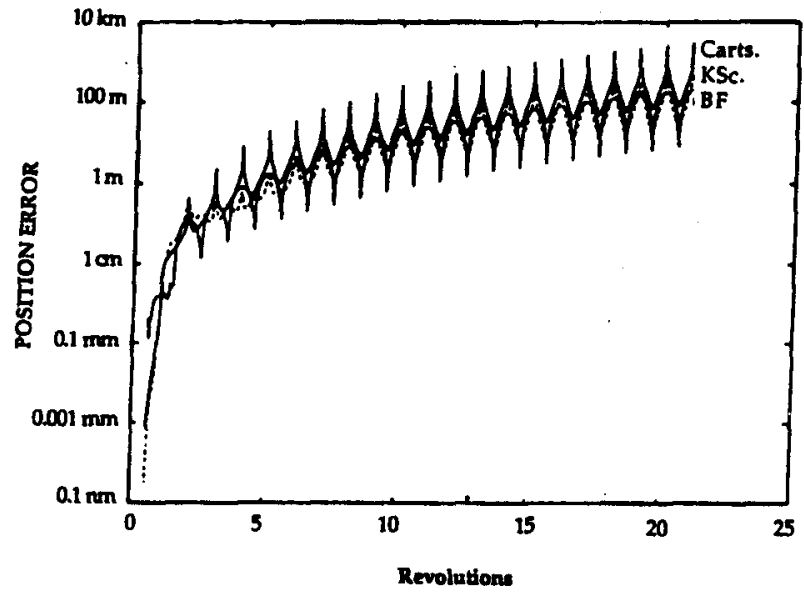

Figure 11 . 
The plots in this figure show once again that the preservation of integrals, although useful, is not a good enough way to check the reliability of the solution obtained by numerically integrating. So, apart from that, we have checked by means of the stabilization of a number of significant digits of several points of the solution distributed along each revolution and backwards integration.

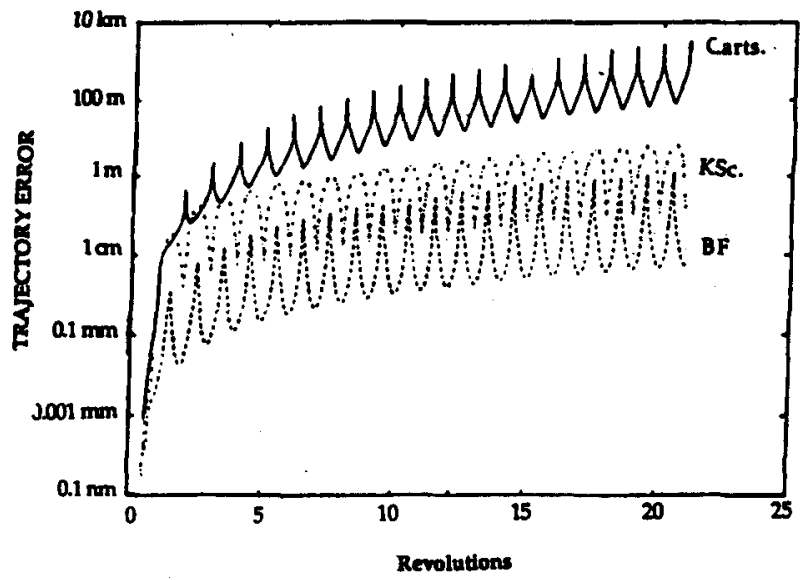

Figure 12 .

\section{ACKNOWLEDGEMENTS}

The authors would like to thank Professor V.Szebehely for his invaluable advise. Also, the authors gratefully acknowledge the financial support of the NATO cooperative research grant $0506 / 88$ and the Spanish CICYT grant ESP88-541.

\section{REF ERENCES}

[1] Aurdet, C.A. (1 969), Le mouvement Keplerien et les oscillateurs harmoniques, J.reine u. angew. Math.,238, 71.

[2] Ferrandiz, J M. (1988), A General Canonical Transformation Increasing the Number of Variables with Application to the Two-Body Problem, Celest. Mech. 41,343.

[3] Ferrandiz, J.M. and Sansaturio, M.E. (1990), Elemento de tiempo en variables de Ferrandiz, Actas XIV JornadasHi spano-Iu sas de Matematicas, Junio 1989 , Vol.III,1231. 
[4] Ferrandiz, J.M., Sansaturio,. M.E. and Pojman, T. (1 990), Increa sed Accuracy of Computations in the Ma in Satellite Problem through Linearization Methods., Celest. Mech. (Shlmitted).

[5] Ferrandiz, J.M.., Sansaturio, M.E. a nd Vigo, J. (1990), On Iong-Time Predictions of satellite Orbits by numerical Integration, in "Predictability, stability and Chaos in N-Body Dynamical Systems", A.E. Roy Ed., Flenum publishing Corporation PATO ASI Series C. (In Press).

[6] Stiefel, E. and Scheifele, G. (1 971), "Linear and Regular Celestial Mechanics", Springer -Verlag, Berlin. 